\title{
Andrea Oberhuber, Corps de papier. Résonances, avec des accompagnements de Catherine Mavrikakis, Nicole Brossard et Verena Stefan
}

Montréal, Nota bene, coll. « Nouveaux Essais Spirale », 2012, $237 \mathrm{p}$.

\section{Evelyne Ledoux-Beaugrand}

Université de Gand

L'ouvrage Corps de papier. Résonances d'Andrea Oberhuber aurait tout aussi bien pu s'intituler « regards sur le corps » tant y est centrale la réflexion sur la manière dont le corps, et le corps féminin en particulier, font toujours l'objet d'une captation visuelle. L'exergue tiré de 58 indices sur le corps de Jean-Luc Nancy pointe déjà dans cette direction. "[L]e 
développement du corps » désigne surtout le long processus de parachèvement d'un sujet dont la finitude ne coïncide qu'avec sa mort, tout en suggérant le façonnement social par lequel le corps se (con)forme. S'entend également dans l'épigraphe la manière dont la corporéité n'est véritablement saisie qu'à travers ses (auto)représentations, ne serait-ce que dans l'image spéculaire réfléchie par le miroir. Car développer le corps réfère implicitement au développement photographique, c'està-dire aux images du corps telles que la photographie notamment les donne à voir. L'inclusion de quelques photos celles d'Unica Zürn, dont les yeux «clair-voyants» (p. 9), comme vus de l'intérieur, viennent encadrer l'essai, et les collages de l'auteure où portraits et dessins se juxtaposent confirme l'idée d'une réflexion sur «la corporéité féminine imaginaire» (p.16) élaborée dans la perspective des regards nombreux posés sur les corps des femmes.

Les points de vues sont en effet décuplés dans cet essai qui répond à un «principe d'enchevêtrement» (p. 17). À travers l'étude d'un «échantillon transhistorique» (p. 15) d'œuvres de femmes, il ne s'agit pas de faire l'histoire de la littérature des femmes depuis l'ère moderne. Bien qu'une mise en perspective historique trace effectivement le chemin de la lecture, les œuvres étudiées ne s'inscrivent dans un rapport ni de convergence thématique ni de progression historique. Elles sont plutôt liées par une semblable «transfrontalité » (p. 108), par un jeu sur les limites du gender et du genre littéraires que poursuit la forme même prise par l'essai d'Andrea Oberhuber. L'hybridité des voix comme des genres caractérise Corps de papier. Ne se contentant pas de multiplier les voix en greffant les contributions de Catherine Mavrikakis, de Nicole Brossard et de Verena Stefan au cœur même de l'ouvrage plutôt qu'en 
appendices, l'auteure opère un dédoublement de sa propre voix et adopte des postures énonciatives diverses relevant tantôt du discours savant, tantôt de la fiction.

\section{Théâtres du corps}

Les œuvres soumises à une analyse orientée par les gender studies appartiennent à trois moments historiques : la charnière des XVIII et XIX ${ }^{\text {e }}$ siècles alors que les premiers balbutiements du romantisme se font entendre dans un contexte encore fortement marqué par la philosophie des Lumières; les années 1930 à 1970, cinq décennies durant lesquelles plusieurs écrivaines-artistes adoptent l'esthétique surréaliste non sans s'inscrire dans un «double mouvement d'affiliation et de désaffiliation » (p.134-135) à ce cercle dirigé d'une main de maître par Breton; et le début du XXI ${ }^{\text {e }}$ siècle, moment où les auteures venues à l'écriture au tournant du siècle sont amenées à se situer par rapport à l'héritage féminin et féministe laissé par leurs prédécesseurs. En tension entre continuité et rupture, obédience au passé et projection vers l'avenir, les romans sentimentaux Ourika et Édouard de Claire de Duras, les œuvres que l'on dirait aujourd'hui intermédiales de Claude Cahun, de Leonora Carrington et de Unica Zürn ainsi que le roman de l'intériorité qu'est La Maison étrangère d'Élise Turcotte ont ceci de commun qu'ils font du corps « une scène de théâtre où les héroïnes de papier tentent d'affronter le destin de ce corps souvent perçu étrangement inquiétant» (p.15) pour des raisons variables d'un récit à l'autre et contingentes des injonctions spécifiques aux époques dont ils sont issus. 
Affirmer, comme le fait Andrea Oberhuber, que les corps féminins, auxquels est accordée une place significative dans les œuvres étudiées, se donnent à la façon d'une « scène de théâtre» (p.188) suppose la conscience, de la part des auteures, d'offrir ces corporéités féminines de papier à l'œil scrutateur d'autrui. Loin d'être simplement contemplatif, ce regard est normatif. Il tient autant du social que de l'imaginaire et est susceptible d'infléchir la trajectoire non seulement des héroïnes mais des créatrices, comme le montre notamment l'analyse des romans de Claire de Duras. Portant un « regard désillusionné » (p. 49) sur ses contemporains, Duras présente des protagonistes résignés à un enfermement dans une solitude volontaire tant ils sont incapables d'échapper aux regards normatifs qui les renvoient à la place que l'époque dit seoir à leur sexe et à leur race. Le sort réservé par la postérité à cette écrivaine précurseur du romantisme, tombée «durant près d'un siècle et demi dans l'un des trous noirs de l'histoire littéraire » (p. 50), n'est pas sans rappeler certains risques et difficultés encourues par celles qui critiquent «les conceptions du genre et des genres littéraires, du corps et des corsets textuels » (p. 14).

La pratique du morcèlement et de la métamorphose par les auteures-artistes surréalistes participe également d'une remise en question de la loi du genre (sexuel et littéraire), pour le dire ici avec Derrida. L'écriture en mosaïque, surtout lorsqu'elle se jumelle avec une pratique artistique comme la peinture, la photographie ou le photomontage, multiplie les points de vues afin d'échapper à une vision restrictive de la «féminité conventionnelle» (p.14). Diffractée, l'image de soi devient insaisissable et même inépuisable, comme l'écrit Oberhuber à propos de la résistance de l'œuvre de Cahun à 
l'exégèse. Pour les héroïnes de Duras, de Cahun, de Carrington et de Zürn, le corps est surtout perçu comme un obstacle qu'il faut soustraire du regard d'autrui en le masquant ou par le biais de constantes métamorphoses et mises en scène. Le corps et la sexualité apparaissent en revanche, pour la protagoniste du roman de Turcotte, comme «deux moyens efficaces de maintenir le lien avec autrui, de rester humain et d'échapper à la solitude à l'état pur » (p. 183), à la condition que ce corps ait été préalablement aménagé, rendu habitable par un travail d'écriture qui révèle comment la corporéité est inextricablement le lieu d'une textualité.

\section{L'autre scène de l'écriture}

Partageant à part égale l'espace du livre avec l'écriture de type analytique, la fiction permet à l'auteure de revisiter les œuvres étudiées sous un nouvel angle. Cette deuxième scène d'écriture et, par extension, de lecture pour les lecteurs et lectrices qui entrent dans l'ouvrage, approche ce que le discours savant, soumis à une obligation de maîtrise, se doit normalement de tenir à distance, à savoir l'inconscient et le corps, ou encore «l'énergie du rêve qui échappe au genre » (p. 72) comme l'écrit Nicole Brossard dans sa contribution à l'essai. S'intercalant entre les propos analytiques, les formes de l'intime choisies par Andrea Oberhuber - quelques pages d'un journal porté par un « je » identifié à Claire de Duras, des lettres adressées à chacune des trois créatrices surréalistes étudiées, et un livre d'heures écrit sous le pseudonyme d'Andrina - contribuent à dévoyer le centre de Corps de papier, à tracer des chemins de traverse où 
s'expriment les «idées que l'on ne veut pas ou ne peut pas exprimer ailleurs » (p.129). S'y révèle un rapport intime aux œuvres et à l'écriture: "Et moi alors, demande une voix intérieure, quel rapport entretiens-je avec l'écriture? Pourraisje un jour me déjouer moi-même, détourner à l'aide d'un paratonnerre littéral le regard critique sagement acquis au cours de mes études? » (p. 197) Si ces incursions du côté du « je » n'écartent pas toute idée de maîtrise, loin s'en faut, et semblent en ce sens bien loin de « ces errances, ces lapsus et ces bégaiements de la réflexion » (p. 23) qui rythment le livre issu de la rencontre entre Marguerite Duras et Xavière Gauthier, il est vrai, comme le suggère Catherine Mavrikakis dans le chapitre "Pour une théâtralité du livre», que Corps de papier s'inscrit dans une certaine parenté avec Les Parleuses "par sa forme, son exigence et sa nouveauté » (p. 24). Par la façon qu'il a de défier et de déjouer les genres sans pour autant donner aux lecteurs et lectrices l'impression de s'égarer.

Caractérisé par une économie de la transfrontalité et par une intermédialité, l'essai échappe en effet au chaos ou au baroque. Dans l'espace de la rencontre qu'il ouvre, Corps de papier demeure cohérent. Si l'écriture se veut une « atteinte à la "peaurosité" des frontières » (p. 205) entre le «je » exposé au regard du monde et le «je » dérobé à celui-ci, cette perméabilité, de la peau comme de l'écriture, rend floue, sans toutefois l'abolir, la frontière entre moi et autrui, entre dedans et dehors, entre essai et fiction. Sans être tout à fait ni à l'extérieur ni à l'intérieur des genres, l'écriture demeure à cheval sur les délimitations, qui s'en trouvent ainsi déplacées. La mise en scène déployée autour du pseudonyme de l'auteure témoigne de cette oscillation constante entre des pôles multiples. Utilisé qu'à l'intérieur des pages du livre et plus 
précisément dans le "Livre d'heures d'Andrina», le nom d'emprunt voile certes l'identité de l'auteure mais c'est pour mieux la dévoiler à travers l'explication de sa provenance. Mais même une fois divulguée, l'histoire du prénom Andrina se place encore sous le signe des transformations qui ont partie liées avec une posture d'énonciation d'exilée située au point de jonction de plusieurs frontières, entre autres linguistiques et géographiques. À l'instar du corps pensé comme un lieu de $\mathrm{f}(\mathrm{r})$ iction entre le sujet et le monde, le « je » démultiplié devient une sorte de carrefour où l'auteure met un peu d'elle-même et un peu des autres, dans un jeu de mise à distance et de reflets digne des écrivaines surréalistes qui lui sont chères. Tout en se faisant poreux aux voix des autres, voire "peaureux» pour reprendre le beau mot-valise proposé par Andrea Oberhuber, le livre aménage l'espace nécessaire à l'émergence d'une parole originale, à la fois singulière et plurielle, qui engage lecteurs et lectrices à envisager le corps sous ses biais matériel, visuel et textuel. 